\title{
FREQUENT HYPERCYCLICITY, CHAOS, AND UNCONDITIONAL SCHAUDER DECOMPOSITIONS
}

\author{
by \\ Manuel De la Rosa, Leonhard Frerick, Sophie Grivaux \& Alfredo Peris
}

\begin{abstract}
We prove that if $X$ is any complex separable infinite-dimensional Banach space with an unconditional Schauder decomposition, $X$ supports an operator $T$ which is chaotic and frequently hypercyclic. In contrast with the complex case, we observe that there are real Banach spaces with an unconditional basis which support no chaotic operator.
\end{abstract}

\section{Introduction}

We are interested in this paper in the dynamics of continuous linear operators acting on a complex infinite-dimensional separable Banach space $X$. If $T$ is such an operator on $X, T$ is said to be hypercyclic if there exists a vector $x \in X$ (a hypercyclic vector for $T$ ) such that $\mathcal{O} \operatorname{rb}(x, T)=\left\{T^{n} x ; n \geq 0\right\}$ is dense in $X$. Hypercyclicity has had many developments in the past years, and we refer the reader to the recent books [5] and [14] for a thorough account of the subject.

We study here reinforcements of hypercyclicity: chaotic operators are topologically transitive operators (or, in other words, hypercyclic operators) which have a dense set of periodic points (a vector $x \in X$ is said to be periodic if there exists an integer $N \geq 1$ such that $T^{N} x=x$ ). This notion of chaos coincides in our setting with the classical one introduced by Devaney. Another strengthening of hypercyclicity is the notion of frequent hypercyclicity, which was introduced in [2]: $T$ is said to be frequently hypercyclic if there exists a vector $x \in X$ such that for every non-empty open subset $U$ of $X$, the set $\left\{n \geq 0 ; T^{n} x \in U\right\}$ of instants when the iterates of $x$ under $T$ visit $U$ has positive lower density:

$$
\liminf _{N \rightarrow+\infty} \frac{1}{N}\left|\left\{n \leq N ; T^{n} x \in U\right\}\right|>0
$$

2000 Mathematics Subject Classification. - 47A16, 37A05, 47A35, 46B09, 46B15.

Key words and phrases. - Linear dynamical systems; hypercyclic, chaotic, and frequently hypercyclic operators; spaces with an unconditional Schauder decomposition.

This work was partially supported by ANR-Projet Blanc DYNOP, by the MEC and FEDER Projects MTM2007-64222 and MTM2010-14909, and by Generalitat Valenciana Project PROMETEO/2008/101. 
where $|A|$ denotes the cardinality of a finite subset $A \subset \mathbb{N}$. It was proved independently by Ansari [1] and Bernal-Gonzalez [6] that any separable infinite-dimensional Banach space $X$ supports a hypercyclic operator. These operators are of the form $T=I+K$, where $K$ is a nuclear backward weighted shift with respect to a biorthogonal system of $X$. In particular the spectrum of $T$ is reduced to the point $\{1\}$. The fact that chaos and frequent hypercyclicity are really stronger notions than hypercyclicity is attested by the fact that the corresponding existence result does not hold true anymore. That is, it was shown by Bonet, Martínez-Giménez and Peris [8] that some Banach spaces do not support any chaotic operator, and by Shkarin [16] that the same spaces do not support any frequently hypercyclic operator. The class of spaces considered in [8] and [16] is the class of complex hereditarily indecomposable Banach spaces (like the space of Gowers and Maurey [12]). We recall that a Banach space $X$ is said to be hereditarily indecomposable if no closed subspace of $X$ is decomposable as a direct sum of infinite-dimensional subspaces. On such spaces every operator has the form $T=\lambda I+S$, where $\lambda$ is a scalar and $S$ is a strictly singular operator on $X$. Hence if $T$ is hypercyclic, the spectrum of $T$ is reduced to the point $\{\lambda\}$ with $|\lambda|=1$. Now it is proved in [16 that the spectrum of a frequently hypercyclic operator cannot have an isolated point, and the same holds true for a chaotic operator.

The main purpose of this work is to investigate the following question:

Question 1.1. - Is it possible to characterize the complex separable Banach spaces which support a frequently hypercyclic (respectively a chaotic) operator?

We are not able to answer completely Question [1.1, but we prove the following theorem, which gives a fairly large class of spaces on which such operators can indeed be constructed:

Theorem 1.2. - Let $X$ be a complex separable Banach space having an unconditional Schauder decomposition. Then $X$ supports an operator which is frequently hypercyclic and chaotic.

In particular any complex Banach space with an unconditional basis admits a frequently hypercyclic and chaotic operator. Actually, it is still an open question to know whether every chaotic operator on a Banach space is automatically frequently hypercyclic; see [13. for more details on this question.

The frequently hypercyclic operators constructed in the proof of Theorem 1.2 have an interesting property: they are compact (even nuclear) perturbations of diagonal operators whose diagonal coefficients are complex numbers of modulus 1 . The proof of Theorem 1.2 is done via a transference argument. In other words, we first construct a class of nuclear perturbations of diagonal operators on a Hilbert space, then transfer these operators to our Banach spaces. We show in fact that our operators enjoy a stronger property:

Theorem 1.3. - If $X$ is a separable complex Banach space which has an unconditional Schauder decomposition, then $X$ supports a bounded operator $T$ which is ergodic with respect to a non-degenerate invariant Gaussian measure.

The Hilbert space construction is carried out in Section 2, and Theorems 1.2 and 1.3 are proved in Section 3. Finally, we show in Section 4 that the situation is drastically different 
if we change the scalar field: there are real Banach spaces with an unconditional basis which support no chaotic operators. The corresponding question for frequent hypercyclicity is still open.

Let us finish this introduction by mentioning that it is possible to extend Theorem 1.2 to the case of Fréchet spaces [9]: every complex separable Fréchet space with a continuous norm and an unconditional Schauder decomposition admits a frequently hypercyclic and chaotic operator. The same result holds true for complex Fréchet spaces with an unconditional basis.

\section{Some frequently hypercyclic and chaotic operators on Hilbert spaces}

So our aim is to show that if $X$ is a separable complex infinite-dimensional Banach space with an unconditional Schauder decomposition, then $X$ supports an operator which is both chaotic and frequently hypercyclic. We begin by constructing a particular class of nuclear perturbations of diagonal operators on a Hilbert space, which consists of frequently hypercyclic and chaotic operators.

For $n \geq 0$, let $H_{n}$ be the space $\ell^{2}$ endowed with the canonical basis $\left(e_{i, n}\right)_{i \geq 0}$, and let $H=\oplus_{\ell^{2}} H_{n}$ be the orthogonal sum of all the spaces $H_{n}$. If $\left(\mu_{n}\right)_{n \geq 0}$ is any bounded sequence of complex numbers, the diagonal operator $\bar{D}_{\mu}$ is defined by $\bar{D}_{\mu}\left(\oplus x_{n}\right)=\oplus \mu_{n} x_{n}$ for any element $x=\oplus x_{n}$ in $H$. Now let $\left(\left(w_{i, n}\right)_{i \geq 0}\right)_{n \geq 0}$ be a sequence of positive weights such that

$$
\sup _{n \geq 0} \sup _{i \geq 0} w_{i, n}<+\infty .
$$

We define the operator $\bar{B}_{w}$ on $H$ by setting

$$
\bar{B}_{w} x=\sum_{n \geq 1} \sum_{i \geq 0}\left\langle x, e_{i, n}\right\rangle w_{i, n-1} e_{i, n-1} .
$$

This is clearly a bounded operator on $H$, which is nothing but a backward weighted shift: $\bar{B}_{w} e_{i, 0}=0$ for any $i \geq 0$, and $\bar{B}_{w} e_{i, n}=w_{i, n-1} e_{i, n-1}$ for $n \geq 1$ and $i \geq 0$.

Theorem 2.1. - Let $w=\left(\left(w_{i, n}\right)_{i \geq 0}\right)_{n \geq 0}$ be a bounded sequence of positive weights. There exists a sequence $\left(\mu_{n}\right)_{n \geq 0}$ of unimodular numbers such that the operator $\bar{T}=$ $\bar{D}_{\mu}+\bar{B}_{w}$ is frequently hypercyclic and chaotic on $H$.

The proof of Theorem 2.1 relies on a criterion for frequent hypercyclicity which was proved in [2] in the Hilbert space setting, and which states that if $T \in \mathcal{B}(H)$ has "sufficiently many" eigenvectors associated to eigenvalues of modulus 1 , then $T$ is frequently hypercyclic. Here is the precise definition:

Definition 2.2. - We say that a bounded operator $T$ on $X$ has a perfectly spanning set of eigenvectors associated to unimodular eigenvalues if there exists a continuous probability measure $\sigma$ on the unit circle $\mathbb{T}$ such that for every $\sigma$-measurable subset $A$ of $\mathbb{T}$ which is of $\sigma$-measure $1, \operatorname{span} \bigcup_{\lambda \in A} \operatorname{ker}(T-\lambda I)$ is dense in $X$.

In other words if we take out from the unit circle a set of $\sigma$-measure 0 of eigenvalues, the eigenvectors associated to the remaining eigenvalues still span $X$. 
The following result is proved in [3]:

Theorem 2.3. - [3] If T is a bounded operator acting on a separable infinite-dimensional complex Hilbert space $H$, and if $T$ has a perfectly spanning set of eigenvectors associated to unimodular eigenvalues, then $T$ is frequently hypercyclic.

See 13 for a different proof which extends to the Banach space case. We are now ready for the proof of Theorem 2.1 .

Proof of Theorem 2.1. - Let $\mu=\left(\mu_{n}\right)_{n \geq 0}$ be for the moment an arbitrary sequence of unimodular numbers. Let $\lambda \in \mathbb{T}$, and $x=\oplus x_{n} \in H$. Then $\bar{T} x=\lambda x$ if and only if for any $n \geq 0$ and any $i \geq 0$,

$$
\mu_{n} x_{n}+\sum_{i \geq 0}\left\langle x_{n+1}, e_{i, n+1}\right\rangle w_{i, n} e_{i, n}=\lambda x_{n}
$$

that is

$$
\left\langle x_{n}, e_{i, n}\right\rangle=\frac{\lambda-\mu_{n-1}}{w_{i, n-1}}\left\langle x_{n-1}, e_{i, n-1}\right\rangle \quad \text { so that } \quad\left\langle x_{n}, e_{i, n}\right\rangle=\prod_{p=0}^{n-1}\left(\frac{\lambda-\mu_{p}}{w_{i, p}}\right) .
$$

Hence provided the series involved are convergent, the vectors

$$
E_{i}(\lambda)=e_{i, 0}+\sum_{n \geq 1}\left(\prod_{p=0}^{n-1} \frac{\lambda-\mu_{p}}{w_{i, p}}\right) e_{i, n}, \quad i \geq 0
$$

are eigenvectors of $\bar{T}$ which span the eigenspace $\operatorname{ker}(\bar{T}-\lambda)$. Now our aim is to construct the diagonal coefficients $\mu_{p}$, and a Cantor set $K \subset \mathbb{T}$ containing the $\mu_{p}$ 's, in such a way that the eigenvector fields $E_{i}, i \geq 0$, are well-defined and continuous on $K$, and the unimodular eigenvectors of $\bar{T}$ are perfectly spanning.

Write the set $\mathbb{N}_{0}$ of nonnegative integers as the disjoint union of successive intervals $J_{k}$, $k \geq 0$, where $\left|J_{k}\right|=2^{k-1}$ for $k \geq 1: J_{0}=\{0\}, J_{1}=\{1\}, J_{2}=\{2,3\}, J_{3}=\{4,5,6,7\}$, $J_{4}=\{8,9,10,11,12,13,14,15\}$, etc... and more generally $J_{k}=\left\{2^{k-1}, \ldots, 2^{k}-1\right\}$.

- Step 0: to begin with, we take $\mu_{0}=1$.

- Step 1: let $\mu_{1}$ be an element of $\mathbb{T}$ distinct from $\mu_{0}$ with $\left|\mu_{0}-\mu_{1}\right|<1$ and such that the length $l_{1}=\left|\mu_{0}-\mu_{1}\right|$ of the closed arc $\Gamma_{1}$ joining in $\mathbb{T} \mu_{0}$ and $\mu_{1}$ is so small that

$$
l_{1}^{2} \sum_{n=2}^{3}\left(\prod_{p=0}^{n-1} \frac{1}{w_{i, p}^{2}}\right)<2^{-3} \quad \text { for } i=0,1 .
$$

- Step k: at step $k$, we construct the unimodular numbers $\mu_{n}$ for $n \in J_{k}$. We choose them in the set $\bigcup_{p \in J_{k-1}} \Gamma_{p}$, where, for $p \in J_{k-1}, \Gamma_{p}$ denotes the closed arc in $\mathbb{T}$ joining $\mu_{p}$ and $\mu_{p-2^{k-2}}$. We also require that $\mu_{0}, \ldots, \mu_{2^{k}-1}$ be all distinct, and that for each $j=0, \ldots, 2^{k-1}-1, \mu_{2^{k-1}+j}$ be very close to $\mu_{j}$. More precisely, we pick these numbers $\mu_{2^{k-1}}, \ldots, \mu_{2^{k}-1}$ in such a way that $l_{k}=\max \left\{\left|\mu_{2^{k-1}+j}-\mu_{j}\right| ; j=0, \ldots, 2^{k-1}-1\right\}$ is so small that

$$
l_{k}^{2} \sum_{n \in J_{k+1}}\left(\prod_{p=0}^{n-1} \frac{1}{w_{i, p}^{2}}\right)<2^{-(k+2)} \quad \text { for } i=0,1, \ldots, k .
$$


We then define $\Gamma_{2^{k-1}+j}$ for $j=0, \ldots, 2^{k-1}-1$ as the closed arc in $\mathbb{T}$ joining $\mu_{j}$ and $\mu_{2^{k-1}+j}$. Let $K$ be the compact subset of $\mathbb{T}$ defined by

$$
K:=\bigcap_{k \in \mathbb{N}}\left(\bigcup_{j \in J_{k}} \Gamma_{j}\right)
$$

By construction, $\mu_{p}$ is in $K$ for every $p \geq 0$. Fix $\lambda \in K$. Since $\lambda$ belongs to $\bigcup_{j \in J_{k}} \Gamma_{j}$ for any $k \geq 1$, there exists for any $k \geq 1$ an integer $q \in J_{k}$ with $\left|\lambda-\mu_{q}\right| \leq l_{k}$. Moreover the diameter of $K$ is smaller than 1 , and thus for every $k \geq 1$ and every $n \geq 2^{k}$, we have

$$
\prod_{p=0}^{n-1}\left|\lambda-\mu_{p}\right|^{2}<l_{k}^{2}
$$

Therefore we have for each $i \geq 0$ and each $k^{\prime} \geq i$

$$
\sum_{k \geq k^{\prime}} \sum_{n \in J_{k+1}}\left(\prod_{p=0}^{n-1} \frac{\left|\lambda-\mu_{p}\right|^{2}}{w_{i, p}^{2}}\right)<\sum_{k \geq k^{\prime}} \sum_{n \in J_{k+1}}\left(l_{k}^{2} \prod_{p=0}^{n-1} \frac{1}{w_{i, p}^{2}}\right)<\sum_{k \geq k^{\prime}} 2^{-(k+2)}=2^{-\left(k^{\prime}+1\right)} .
$$

This implies that the series defining $E_{i}(\lambda)$ converges for every $\lambda \in K$ and every $i \geq 0$.

Let us now prove that the eigenvector field $E_{i}$ is continuous on $K$ for each $i \geq 0$. For any $\varepsilon>0$, let $k_{0} \geq i$ be such that $2^{-k_{0}}<\varepsilon / 2$, and let

$$
P(\lambda)=e_{i, 0}+\sum_{n=1}^{2^{k_{0}}}\left(\prod_{p=0}^{n-1} \frac{\lambda-\mu_{p}}{w_{i, p}}\right) e_{i, n}
$$

be the polynomial in $\lambda$ corresponding to the $2^{k_{0}}$-th partial sum in the expression of $E_{i}$. Then for all $\lambda, \lambda^{\prime} \in K$ we have

$$
\left\|E_{i}(\lambda)-E_{i}\left(\lambda^{\prime}\right)\right\|^{2}<\left\|P(\lambda)-P\left(\lambda^{\prime}\right)\right\|^{2}+2.2^{-\left(k_{0}+1\right)}<\left\|P(\lambda)-P\left(\lambda^{\prime}\right)\right\|^{2}+\frac{\varepsilon}{2} .
$$

Let now $\delta>0$ be such that $\left\|P(\lambda)-P\left(\lambda^{\prime}\right)\right\|^{2}<\varepsilon / 2$ whenever $\left|\lambda-\lambda^{\prime}\right|<\delta$. Then

$$
\left\|E_{i}(\lambda)-E_{i}\left(\lambda^{\prime}\right)\right\|^{2}<\varepsilon
$$

for any $\lambda, \lambda^{\prime} \in K$ with $\left|\lambda-\lambda^{\prime}\right|<\delta$, and this proves that the eigenvector fields $E_{i}, i \geq 0$, are continuous on $K$.

Observe now that the construction is done in such a way that all the coefficients $\mu_{p}$ are distinct. This implies that the eigenvectors $E_{i}(\lambda), i \geq 0, \lambda \in K$, span a dense subspace of $H$. Indeed for any $N \geq 0, \mu_{N}$ belongs to $K$ by construction, and

$$
E_{i}\left(\mu_{N}\right)=e_{i, 0}+\sum_{n=1}^{N}\left(\prod_{p=0}^{n-1} \frac{\mu_{N}-\mu_{p}}{w_{i, p}}\right) e_{i, n}
$$

Hence $\operatorname{sp}\left[E_{i}\left(\mu_{N}\right) ; i, N \geq 0\right]$ contains all the vectors $e_{i, n}, i \geq 0, n \geq 0$. By continuity of the eigenvector fields $E_{i}$, if $\sigma$ is any continuous measure whose support is $K$, the eigenvectors $E_{i}(\lambda), i \geq 0, \lambda \in K$, are perfectly spanning with respect to $\sigma$. Such a measure $\sigma$ exists because $K$ is a perfect compact subset of $\mathbb{T}$. Since our operator $\bar{T}$ is living on a Hilbert space, Theorem 2.3 can be applied and $\bar{T}$ is frequently hypercyclic. 
If we additionally require that $\bar{T}$ be chaotic, it suffices to choose all the coefficients $\mu_{p}$ to be $n^{\text {th }}$ roots of 1 . Then the eigenvectors of $\bar{T}$ associated to eigenvalues which are $n^{\text {th }}$ roots of 1 span a dense subspace of $H$, and $\bar{T}$ is chaotic.

\section{Proofs of Theorems 1.2 and 1.3}

3.1. Unconditional Schauder decompositions. - Let $X$ be a separable infinitedimensional Banach space which admits an unconditional Schauder decomposition. This means that there exists a sequence $\left(X_{n}\right)_{n \geq 0}$ of closed subspaces of $X$ such that any $x \in X$ can be written in a unique way as an unconditionally convergent series $x=\sum_{n \geq 0} x_{n}$, where $x_{n}$ belongs to $X_{n}$ for any $n \geq 0$. We denote in this case by $P_{n}$ the canonical projection $x \mapsto x_{n}$ of $X$ onto $X_{n}$. If $\left(X_{n}\right)_{n \geq 0}$ is an unconditional Schauder decomposition of $X$, and $\left(I_{k}\right)_{k \geq 0}$ is any partition of $\mathbb{N}$ into finite or infinite subsets, let $Y_{k}$ denote the closed linear span of the spaces $X_{n}, n \in I_{k}$. Then $\left(Y_{k}\right)_{k \geq 0}$ is also an unconditional Schauder decomposition of $X$. Hence we will always suppose in the sequel that $\left(X_{n}\right)_{n \geq 0}$ is an unconditional Schauder decomposition of $X$ with all the subspaces $X_{n}$ infinite-dimensional. If $\left(\mu_{n}\right)_{n \geq 0}$ is any bounded sequence of complex numbers, then the multiplication operator $D_{\mu}: X \rightarrow X$ defined by

$$
D_{\mu}\left(\sum_{n \geq 0} x_{n}\right)=\sum_{n \geq 0} \mu_{n} x_{n}
$$

is a bounded operator on $X$ since the decomposition $X=\oplus_{n \geq 0} X_{n}$ is unconditional. Now since all $X_{n}$ 's are infinite-dimensional, each of them admits a biorthogonal system $\left(x_{i, n}, x_{i, n}^{*}\right)_{i \geq 0}$, where $x_{i, n} \in X_{n}, x_{i, n}^{*} \in X_{n}^{*}$ and $\left\langle x_{i, n}^{*}, x_{j, n}\right\rangle=\delta_{i j}$ for $i, j \geq 0$. Since $X=X_{n} \oplus \overline{\operatorname{span}} \bigcup_{p \neq n} X_{p}$, we can extend $x_{i, n}^{*}$ to $X$ by setting $x_{i, n}^{*}=0$ on $\overline{\operatorname{span}} \bigcup_{p \neq n} X_{p}$. For $n \geq 1$, let $\left(w_{i, n}\right)_{i \geq 0}$ be a sequence of positive weights going to zero very fast when $i$ goes to infinity. Denote by $w$ the collection of sequences $\left(\left(w_{i, n}\right)_{i \geq 0}\right)_{n \geq 1}$, and define the operator $B_{w}$ on $X$ by setting

$$
B_{w} x=\sum_{n \geq 1} \sum_{i \geq 0}\left\langle x_{i, n}^{*}, x\right\rangle w_{i, n} x_{i, n-1}=\sum_{n \geq 1} \sum_{i \geq 0}\left\langle x_{i, n}^{*}, P_{n} x\right\rangle w_{i, n} x_{i, n-1} .
$$

Let $\|\cdot\|^{*}$ be the dual norm of $\|\cdot\|$. If the series

$$
\sum_{n \geq 1} \sum_{i \geq 0} w_{i, n}\left\|x_{i, n-1}\right\| \cdot\left\|x_{i, n}^{*}\right\|^{*}
$$

is convergent (which is the case if the quantities $w_{i, n}$ are suitably small), $B_{w}$ is a nuclear operator, hence a bounded operator.

3.2. Proof of Theorem 1.2. - The proof of Theorem 1.2 is now a straightforward application of the transference principle, as applied for instance in [1]. Without loss of generality we can suppose that

$$
\sum_{n \geq 0}\left(\sum_{i \geq 0}\left\|x_{i, n}\right\|^{2}\right)<+\infty .
$$


Then the operator $J: H \longrightarrow X$ defined by $J e_{i, n}=x_{i, n}$ for $i, n \geq 0$ is bounded, injective, and has dense range. Let $\left(\left(w_{i, n}\right)_{i \geq 0}\right)_{n \geq 0}$ be a bounded sequence of positive weights such that the operator $B_{w}$ defined above is bounded on $X$. If $\bar{B}_{w}$ denotes the backward weighted shift on $H$ associated to $w$, then $J \bar{B}_{w}=B_{w} J$. Now by Theorem 2.1 there exists a sequence of unimodular numbers such that $\bar{T}=\bar{D}_{\mu}+\bar{B}_{w}$ is frequently hypercyclic and chaotic on $H$. Then $J\left(\bar{D}_{\mu}+\bar{B}_{w}\right)=\left(D_{\mu}+B_{w}\right) J$, and since $J$ is injective and has dense range, $T$ is frequently hypercyclic and chaotic on $X$.

3.3. Ergodicity with respect to an invariant gaussian measure: proof of Theorem 1.3. - The proof of Theorem 1.3 is an immediate consequence of the intertwining equation $J\left(\bar{D}_{\mu}+\bar{B}_{w}\right)=\left(D_{\mu}+B_{w}\right) J$ above. Since $\bar{T}$ acts on a Hilbert space, $\bar{T}$ admits an ergodic non-degenerate invariant Gaussian measure $\bar{m}$ by [3]. Let $m$ be the Gaussian measure on $X$ defined by $m(A)=\bar{m}\left(J^{-1}(A)\right)$ for any Borel subset $A$ of $X$. This measure is non-degenerate and invariant by $T$. Lastly it is not difficult to check that $T$ is ergodic with respect to $m$ : if $m(A)>0$ and $m(B)>0$, there exists an integer $N$ such that $\bar{m}\left(\bar{T}^{-N}\left(J^{-1}(A)\right) \cap J^{-1}(B)\right)>0$. Since $J^{-1}\left(T^{-N}(A) \cap B\right)=\bar{T}^{-N}\left(J^{-1}(A)\right) \cap J^{-1}(B)$ by the intertwining equation $T J=J \bar{T}$, we have $m\left(T^{-N}(A) \cap B\right)>0$. This shows that $T$ is ergodic with respect to $m$.

\section{Chaotic operators and unconditional basis: the real case}

In this section, we observe that the situation changes completely if instead of considering complex Banach spaces as in Section 3, we consider real Banach spaces.

The counterexample of Theorem 4.1 below is built on a real Banach space $X_{G}$ constructed by Gowers in [10]: $X_{G}$ has an unconditional basis $\left(e_{n}\right)_{n}$, and every bounded operator $T \in L\left(X_{G}\right)$ is of the form $T=D_{a}+S$, where $D_{a}$ is a diagonal operator with respect to the basis $\left(e_{n}\right)_{n}$ associated to a bounded weight $a=\left(a_{n}\right)_{n} \in \mathbb{R}^{\mathbb{N}}$, and $S$ is a strictly singular operator (see [11]).

Theorem 4.1. - The real separable infinite-dimensional Banach space $X_{G}$ has an unconditional basis, but admits no chaotic operator.

Proof. - Suppose that $T \in \mathcal{B}\left(X_{G}\right)$ is a chaotic operator on $X_{G}, T=D_{a}+S$, where $D_{a}$ is the diagonal operator with respect to the unconditional basis $\left(e_{n}\right)_{n}$ corresponding to $a=\left(a_{n}\right)_{n} \in \mathbb{R}^{\mathbb{N}}$. Then its complexification $\widetilde{T}$ on the complexification $\widetilde{X}_{G}$ is also chaotic 7], and $\widetilde{T}$ can be written as $\widetilde{T}=\widetilde{D}_{a}+\widetilde{S}$. Since $\widetilde{T}$ is chaotic, its spectrum has no isolated points and intersects $\mathbb{T} \backslash \mathbb{R}$. Therefore we can select a boundary point $\lambda$ of $\sigma(\widetilde{T}) \backslash \mathbb{R}$ and, by Putnam's theorem (see, e.g., Proposition 3.7.8 in [15]) we obtain that $\lambda$ belongs to the essential spectrum of $\widetilde{T}$. On the other hand, since every $a_{n}$ belongs to $\mathbb{R}$, the operator $\widetilde{D}_{a}-\lambda I$ is invertible on $\widetilde{X}_{G}$. Thus $\widetilde{T}-\lambda I$, being a perturbation of an invertible operator by a strictly singular operator, is a Fredholm operator of index 0 on $\widetilde{X}_{G}$, which contradicts the fact that $\lambda \in \sigma_{e}(\widetilde{T})$. Hence $T$ is not chaotic on $X_{G}$.

Theorem 4.1 leads naturally to the following question: 
Question 4.2. - Does the space $X_{G}$ support a frequently hypercyclic operator? More generally, does there exist a real separable Banach space with an unconditional basis which supports no frequently hypercyclic operator?

Acknowledgement: We would like to thank P. Tradacete for interesting discussions about the results in Section 4 .

\section{References}

[1] S. Ansari, Existence of hypercyclic operators on topological vector spaces, J. Func. Anal. 148 (1997), $384-390$.

[2] F. Bayart and S. Grivaux, Frequently hypercyclic operators, Trans. Amer. Math. Soc. 358 (2006), $5083-5117$.

[3] F. Bayart and S. Grivaux, Hypercyclicity and unimodular point spectrum, J. Funct. Anal. 226 (2005), 281-300.

[4] F. Bayart and S. Grivaux, Invariant Gaussian measures for linear operators on Banach spaces and linear dynamics, Proc. London Math. Soc. 94 (2007), $181-210$.

[5] F. Bayart and É. Matheron, Dynamics of linear operators, Cambridge University Press, Vol. 179 (2009).

[6] L. Bernal-González, On hypercyclic operators on Banach spaces, Proc. Amer. Math. Soc., 127 (1999), 1003 - 1010.

[7] J. BÈs And A. Peris, Hereditarily hypercyclic operators, J. Funct. Anal. 167 (1999), 94-112.

[8] J. Bonet, F. Martínez-Giménez and A. Peris, A Banach space wich admits no chaotic operator, Bull. London Math. Soc., 33 (2001), 196 - 198.

[9] M. De la Rosa, L. Frerick, S. Grivaux and A. Peris, Unconditional decompositions, frequent hypercyclicity and chaos for operators on Fréchet spaces, preprint 2010.

[10] W. T. Gowers, A solution to Banach's hyperplane problem, Bull. London Math. Soc. 26 (1994), 523-530.

[11] W. T. Gowers and B. Maurey, Banach spaces with small spaces of operators, Math. Ann. 307 (1997), 543-568.

[12] W. T. Gowers And B. Maurey, The unconditional basic sequence problem, J. Amer. Math. Soc., 6 (1993), $851-874$.

[13] S. Grivaux, A new class of frequently hypercyclic operators, to appear in Indiana Univ. J. Math.

[14] K. G. Grosse-Erdmann and A. Peris, Linear Chaos, Universitext, Springer, to appear.

[15] K. B. Laursen and M. M. Neumann, An Introduction to Local Spectral Theory, London Mathematical Society Monographs, New Series 20, Oxford: Clarendon Press, 2000.

[16] S. Shkarin, On the spectrum of frequently hypercyclic operators, Proc. Amer. Math. Soc. 137 (2009), $123-134$.

May 9, 2018 
Manuel De la Rosa, Institut Universitari de Matemàtica Pura i Aplicada (IUMPA), Universitat Politècnica de València, 46022 València, Spain • E-mail : madela19@upvnet.upv.es

LeOnhard Frerick, FB IV Mathematik, Universität Trier, D54286 Trier, Germany E-mail : frerick@uni-trier.de

Sophie Grivaux, Laboratoire Paul Painlevé, UMR 8524, Université Lille 1, Cité Scientifique, 59655 Villeneuve d'Ascq Cedex, France • E-mail : grivaux@math.univ-lille1.fr

Alfredo Peris, IUMPA, Universitat Politècnica de València, Departament de Matemàtica Aplicada, Edifici 7A, 46022 València, Spain • E-mail : aperis@mat.upv.es 